HStud 25 (2011)1, 127-142 DOI: 10.1556/HStud.25.2011.1.11

\title{
DAS PRESSBURGER FRAG- UND KUNDSCHAFTSAMT DES ANTON MARTIN, 1781-1783 ${ }^{1}$
}

\author{
ANTON TANTNER ${ }^{2}$ \\ Universität Wien \\ Wien, Österreich
}

\begin{abstract}
Der Artikel beschäftigt sich mit dem im März 1781 von Anton Martin in Pressburg gegründeten Frag- und Kundschaftsamt. Einrichtungen dieser Art waren in habsburgischen Städten seit Beginn des 18. Jahrhunderts geschaffen worden; es handelte sich dabei um Adressbüros, die der Vermittlung von Waren, Arbeitsplätzen, Immobilien und Kapital dienen sollten. Im speziellen Pressburger Fall war damit auch eine Leihbibliothek verbunden und weiters wurde der in dieser Gegend praktizierte Kindertausch zum gegenseitigen Spracherwerb unterstützt. Das Fragamt veröffentlichte auch ein Intelligenzblatt mit dem Namen Preßburger Kundschaftsblatt, scheint aber trotz des weiten Spektrums an angebotenen Dienstleistungen nur auf wenig Resonanz gestoßen zu sein; 1783 musste Martin es schließen. Grundlage des Beitrags sind vorwiegend die in der Universitätsbibliothek Bratislava (Univerzitná knižnica v Bratislave) und in der Kathedralbibliothek Esztergom (Főszékesegyházi Könyvtár) aufbewahrten Ausgaben des Preßburger Kundschaftsblatts.
\end{abstract}

Schlüsselwörter: Adressbüro, Fragamt, Informationsvermittlung, Intelligenzblatt, Kundschaftsblatt, Anton Martin, Pressburg, Ungarn

Im 18. Jahrhundert wurden in den großen Städten der Habsburgermonarchie so genannte Frag- und Kundschaftsämter gegründet; dabei handelte es sich um nach Pariser und Londoner Vorbild geschaffene Adressbüros, ${ }^{3}$ das heißt zumeist mit einem Privileg versehene Einrichtungen, die dem Informationsaustausch dienen sollten: Ihre Aufgaben bestanden in Waren-, Arbeits-, Immobilien- sowie Kapitalvermittlung; wer zum Beispiel ein Gut verkaufen wollte oder Arbeit als Dienstbote suchte, konnte in einem solchen Amt sein oder ihr Begehren gegen Bezahlung einer Einschreibgebühr in ein Protokoll eintragen lassen. Umgekehrt gaben die Bediensteten des Amts gegen Gebühr Auskunft aus diesem Protokoll; in der Regel wurden die Registereinträge auch in einem Anzeigenblatt publiziert, das als Kundschaftsblatt bezeichnet wurde. Je nach Beschaffenheit der städtischen Bedürfnisse setzten die Fragämter unterschiedliche Schwerpunkte in ihrer Tätigkeit; gemeinsam ist ihnen, dass sie als Suchmaschinen avant la lettre betrachtet werden können. ${ }^{4}$ 
In Wien war ein derartiges Fragamt gemeinsam mit dem Versatzamt - dem heutigen Dorotheum - bereits 1707 installiert worden; ab 1721 kooperierte es eng mit dem Wienerischen Diarium, der späteren Wiener Zeitung und ging um 1810 endgültig in dieser auf. Prag und Brünn folgten Mitte des 18. Jahrhunderts, Lemberg 1782, Graz 1783, Innsbruck 1798. ${ }^{5}$ Das erste ungarische Frag- und Kundschaftsamt wurde am 28. März 1781 errichtet; es war von der ungarischen Statthalterei bewilligt worden und als sein Leiter fungierte ein gewisser Anton Martin. ${ }^{6}$ Angekündigt wurde es u. a. durch eine Beilage zur Wiener Zeitung; dieses mit 9. April 1781 datierte Avertissement ${ }^{7}$ versprach, dass die neue Einrichtung nicht nur dasigen Einwohnern, sondern auch jeder auswärtigen Parthey zum gleichmässigen Nutzen und Bequemlichkeit diene [n] sollte und für seine Dienste nur sehr geringe Taxen verlangen würde, womit es dem hohen so als dem niedern bequem und nützlich wäre, von Vorteil für jeden Bürger, Landwirth, Fabrikant, Handelsmann, Künstler, Handwerker, [und] sogar de[m] gemeine[n] Bauersmann.

Nicht weniger als zwölferlei Protokolle sollten in diesem Fragamt geführt werden: Das Kapitalienbuch sollte der Geldvermittlung dienen, wobei je nach Höhe der vermittelten Summe eine verschieden hohe Gebühr zu entrichten war; sechs verschiedene Protokolle waren für die Arbeitsvermittlung gedacht: Das Herrschafts-Haus-Offizierenbuch sollte DienstbotInnen und Herrschaften bzw. landwirtschaftliche Betriebe zusammenbringen, auch sollte ein eigenes Livrey-Leuten-Buch geführt werden, eines für Lehrmeister, also Lehrer, die Lesen, Schreiben, Rechnen, Sprachen, aber auch Musik, Tanz, Reiten und Fechten unterrichten sowie ein Lehrjungen-Buch und ein Buch der conventionirten Wirthschafts-Leuten, worunter Martin dienstsuchende (...) Bräuer, Wirthe, Schäfler, Mayer, Weinzierl, oder sogenannte Beständler: item Kunst-Zier- und Obstgärtner verstand. Ein weiteres Protokoll wurde für theils in keiner Kondition stehende, theils aber aus einer in die andere zu übergehen gesonnene Apotheken-Buchdruckerey-und Freykünstler-Subjekten, dann Kauf- und Handlungsbediente geführt. Für die Eintragung in die Protokolle und die Auskunft darauf verlangte Martin eine Gebühr zwischen 3 und 10 Kreuzer. Spezialität des Pressburger Fragamts war das Buch für Tausch- und Kostkinder, womit ein Protokoll gemeint war, mit dessen Hilfe Kinder zum Fremdsprachenerwerb für eine bestimmte Zeit in eine anderssprachige Familie geschickt werden sollten, eine Praxis, die in dieser ungarisch-, slowakisch- und deutschsprachigen Region schon seit mehreren Jahrhunderten üblich war; die Gebühr dafür betrug 6 Kreuzer. ${ }^{8}$ Für die Immobilienvermittlung war das universal Arenden-Buch gedacht und schließlich gab es drei Protokolle, die der Vermittlung von Waren dienen sollten: Ein fertiger Arbeiten-Buch richtete sich an Handwerker, die Martin dazu aufrief, eine Beschreibung ihrer Produkte mitsamt Angabe des Preises an das Fragamt einzuschicken; mit Hilfe des Naturalienund Materialien-Buch[s] wiederum konnten Nahrungsmittel, Greißlerwaaren 
und Holz verkauft werden, während zu guter Letzt das Fuhrwerk- und Requisiten-Buch Pferde, Wägen, Schlitten und Fässer vermitteln sollte.

Martin garantierte seinen KlientInnen Diskretion; niemand sollte in die Protokolle Einsicht haben und erst bei Zustandekommen eines Vermittlungsakts würde der Namen einer Partei dem andern in das Geschäft Einfluß habenden Theile eröfnet werden.

Weiters kündigte der Fragamtsdirektor an, dass wöchentlich ein Kundschaftsblatt mit Auszügen aus den Protokollen des Amts erscheinen sollte. ${ }^{9}$ Das Exemplar war zunächst um vier, dann um drei Kreuzer zu haben. ${ }^{10}$ Wer das Blatt abonnieren wollte, hatte dafür jährlich zwei Gulden zu begleichen. Dieser Betrag erhöhte sich auf 4 Gulden 18 Kreuzer, sofern das Blatt auswärts per Post verschickt wurde, ${ }^{11}$ tatsächlich rechnete Martin damit, dass es auch InteressentInnen außerhalb Pressburgs gab und rief seine AbonnentInnen dazu auf, ihre Bekannten auf dem Land von den Vorteilen des Kundschaftsblatts zu überzeugen. ${ }^{12}$ In einer späteren Ausgabe gab er an, dass das Blatt auch in die österreichischen Länder, nach Böhmen, Mähren und Schlesien sowie in das Reich verschickt wurde, ${ }^{13}$ wobei er insgesamt allerdings über einen Mangel an Pränumeranten klagte. ${ }^{14}$ Das Kundschaftsblatt bot InteressentInnen auch die Möglichkeit an, gegen Bezahlung beliebige Nachrichten - sofern sie denn erlaubt waren - veröffentlichen zu lassen; ${ }^{15}$ gelehrte Aufsätze und Vorschläge, die den LeserInnen von Nutzen sein konnten, konnten kostenlos publiziert werden, je nach Wunsch mit oder ohne Namen des Verfassers. ${ }^{16}$

Das Pressburger Fragamt kooperierte mit Partnern in Wien und in Pest; so verkündete es seinen LeserInnen, dass es mit zwei in diesen Städten befindlichen Freunden zweimal wöchentlich korrespondieren würde; wer immer etwas dort einkaufen lassen wolle, bräuchte dies nur mitzuteilen und würde promptest bedient. So könnten sich InteressentInnen aus Pest die geschmacktesten Wasser-Melonen, dann Blatter-Toback von dem bekannten Fleischhacker Franz, (...) gemahlene[n] Paprika, (...) türkische[n] Pfeffer, Luft geselchte[n] Speck, gute[n] Ofner Wein, dann die so beliebte Debreziner Saife in ganzen, halben, und Viertel-Tafeln zukommen lassen. Weiters würde das Pressburger Kundschaftsblatt auch nach Pest verschickt werden und könnte beim Sänftenunternehmer - dem Besteller der alldortigen Trage-Sesseln - gegen Gebühr von einem Gulden jährlich gelesen werden. Martin bezeichnete diese Außenstelle als Pester Unter-Amt, das für das Oberam [t] auch Aufträge zur Einschaltung im Pressburger Kundschaftsblatt annehmen würde. ${ }^{17}$ Doch in Pest wurden nicht nur Informationen entgegengenommen: Der Sänftenunternehmer war auch dazu bereit, Wassermelonenkerne gegen Bargeld anzukaufen. ${ }^{18}$ Was die Kooperation mit Wiener Interessenten anbelangt, so lassen sich dafür zwei Beispiele nachweisen: So veröffentlichte das Pressburger Fragamt im Spätsommer 1782 den Auftrag, von Wien aus Pressburger Granit zu verkaufen; ${ }^{19}$ knapp danach bekam es Ordre, für eine Wiener Partei Wermut so- 
wie Wein anzukaufen. Wer solchen anzubieten hätte, sollte eine Probe davon samt Preis an das Fragamt einsenden, das solche an die Wiener Auftraggeber weiterschicken würde. ${ }^{20}$

Der Umfang des Kundschaftsblatts betrug zunächst vier Seiten, ein Raum, der laut Martin oft nicht ausreichte, um die Qualifikationen der Arbeitssuchenden, die zu verkaufenden Waren oder zu vermietenden Wohnungen zu beschreiben, weswegen er seine LeserInnen dazu aufforderte, im Amt wegen genauerer Angaben nachzufragen. ${ }^{21}$ Zumindest anfangs wurden die zu vermittelnden Objekte in einer recht ungeordneten, an eine chinesische Enzyklopädie borges'scher Provenienz gemahnenden Zusammenstellung abgedruckt:

\begin{abstract}
Ein Fechtmeister wird gesucht für einen Cavalier. Item ein Spieltisch von türkischen Haselholz, ein Bedienter, so Frauenzimmer frisiren, und Tafel serviren kann. Ein Heuboden auf 10 Klafter, eine Schupfen auf 6 Wägen, und einige Klafter Holz. Ein Husar, so frisiren, und barbieren kann. Eine Wohnung etwa pr. 2 Zimmer im 1ten Stock. Ein anderes Viertel Jahr Zimmer im 1ten oder 2ten Stock mit Aussicht auf die Gassen. Ferners die im vorigen Wochenblatt erwehnte Arbeits-Leute in einen herrschaftlichen Holzschlag. Nicht minder an dürren Obst: 100 Metzen verschiedene Zwetschgen, 1500 Metzen Nüssen, 100 Metzen Kletzen, mit dem Beysatz, daß der Käufer, wann das gante Quantum nicht von einer Hand zu bekommen wäre, sich mit mehreren Partheyen einlassen, Kontrakte errichten, auch mit der Lieferung bis auf künftigen Herbst warten wolle. Es sind auch Liebhabere, welche eine gewisse Anzahl von sogenannten Ziegelkäs kaufen wollen, welcher auf gräflich Forgacsischen Güttern gemacht wird, und dem Lüneburger-Käs gleich kömmt. Das Amt bittet um Nachricht, und ist bereit solchen an Mann zu bringen. ${ }^{22}$
\end{abstract}

Gemäß dem Avertissement sollte die Hälfte der vom Fragamt geführten Protokolle der Arbeitsvermittlung dienen; Martin schien aber mit Akeptanzproblemen sowohl seitens der Arbeitssuchenden als auch seitens der ArbeitgeberInnen zu kämpfen. So rief er sein Publikum eigens dazu auf, ihm allenfalls vorkommende Dienstlose Oesterreicher, oder sonst Oberländer Hausknechte (...) zuzuschicken $^{23}$ und bedauerte, dass viele, die mittels Fragamt ihr Glück machen könnten, die Güte dieses Amtes nicht einsehen woll[t]en, und sich für die ämtliche Einschreibung gleichsam scheuen würden. ${ }^{24}$ Bei manchen hätte sich das Vorurtheil eingeschlichen, dass ihnen eine Einschreibung präjudicirlich wäre oder dass nur jene sich vormerken ließen, die sich schon nicht mehr selbst helfen könn[t]en, oder wohl gar nicht von guten Kaliber wären. ${ }^{25}$ Dabei gäbe es immer wieder eine Nachfrage nach Arbeitskräften, die leider nicht bedient werden könnte, da sich die Dienstboten nicht meldeten, auch würden öfters Lehrherren vergeblich nach Lehrjungen suchen. ${ }^{26}$ Letztere würden, wenn sie arm wären, unentgeltlich verzeichnet, während Bemittelten für die Einschreibung nicht mehr als 3 Kreuzer 
verrechnet würden; die Eltern der potenziellen Lehrlinge sollten sich eine solche Gelegenheit nicht entgehen lassen. ${ }^{27}$ Die ArbeitgeberInnenseite wurde ebenfalls zu mehr Aktivität animiert: [D]as Fragamt wünscht nur, daß Herrschaften so dergleichen [nämlich arbeitsame Dienstleute] brauchen, sich melden, da sie alsdann jederzeit die täuglichsten auslesen können. ${ }^{28}$ Immerhin würde er das gnädigste Zutrauen einiger hohen Herrschaften genießen, die bereits einige Subjecten durch den Kanal dieses Amtes aufzunehmen geruhet haben, und mit beyderseitiger Zufriedenheit noch dato beybehalten. ${ }^{29}$ Im dritten Jahr seiner Geschäftstätigkeit konnte er schließlich zufrieden feststellen, dass einige herrschaftliche Familien dazu bereit waren, ihre Dienstboten exklusiv durch das Fragamt aufzunehmen. ${ }^{30}$

Zunächst schien Martin daran gedacht zu haben, nur an Männer Arbeit zu vermitteln, als aber auch Frauen diese Dienstleistung in Anspruch nehmen wollten, war er dazu bereit, für diese ein eigenes Protokoll zu eröffnen. ${ }^{31}$ Er betonte allerdings, dass nur derley Dienstsuchende Weibspersonen eingeschrieben würden, die, sofern sie noch nicht in Dienst gewesen wären, hübsche Eltern hätten, die für sie bürgen könnten oder aber Empfehlungsschreiben von angesehenen Personen vorweisen könnten. Von denjenigen Dienstbotinnen, die bereits beschäftigt gewesen waren, verlangte Martin Dienstzeugnisse, die im Amt deponiert werden sollten. ${ }^{32}$ Von der ersten Frau, die auf eine solche Weise Arbeit suchte, sprach der Fragamtsdirektor als von einer wohlgewachsenen Blondine, einer 26jährigen Witwe, die Französisch-, Italienisch- und Deutschkenntnisse vorzuweisen und deren Mann in Diensten eines angesehenen ungarischen Haushalts gestanden hätte. Sie suchte eine Beschäftigung als Kammerfrau oder Gouvernante; weiters boten noch eine Kammerjungfer und zwei Stubenmädchen ihre Dienste an. ${ }^{33}$ Wiederholt tauchten in der Folge beim Fragamt Frauen auf, die mittels seiner Hilfe Arbeit zu finden erhofften: So wies das Kundschaftsblatt auf Frauenzimmer hin, die es verstanden, Preßburger Hauben zu heften und putzen sowie Seidenstrümpfe zu waschen; diese Arbeiterinnen würden unentgeltlich vermittelt werden. ${ }^{34} \mathrm{Au}-$ Berdem annoncierte eine Augenheilerin ihre Künste: Böse Augen oder ein Fell könnte sie von solchen (...) vertreiben; ihre Mittel bestünden blos in einem ganz unschuldigen Wasser, welches für Hitz und kühle Flüsse dienet. ${ }^{35}$

Ein besonderes Service konnte Martin im Frühjahr 1782 anbieten: Es hatte sich bei ihm jemand gemeldet, der Stoffe, Kleider, Vorhänge und Sofaüberzüge färben und auch wieder so waschen konnte, dass die Farbe nicht ausging. Das Fragamt diente als Schnittstelle zu dieser Person: Jeden Montag könnten die zu färbenden oder waschenden Textilien dort abgegeben werden und wären am darauf folgenden Samstag wieder zur Abholung bereit; das Fragamt mutierte somit auch zu einer Wäschereifiliale. ${ }^{36}$ Weiters vermittelte es Schreibarbeiten, da es einen geschickte[n] Menschen bei Handen hatte, der bereit wäre, gegen geringe Bezahlung auf Deutsch und Latein alle Gattungen Schriften aufzusetzen, und auch sehr 
sauber in das Reine zu bringen (...), es mögen nun Bittschriften, Kontrakten, Briefe, Auszügeln, Lehrbriefe, Berechnungen, Schuldscheine, Visit- Tafel- musikalische Akademien, und Ball-Bilieten, verzierte Tittl-Blätter, Innschriften auf gezeichnete Riße oder Plane, dann Münz oder Medaillen, und Naturalien Kabinete, oder was immer seyn. Im Gegensatz zu den Winkelschreibern, die nur Verwirrungen stiften und durch die viele Personen um ihr Geld gebracht würden, könnten sich die AuftraggeberInnen der dem Schreiber anvertrauten Dokumente sicher sein und auch darauf vertrauen, dass dieser angesichts der in den Schriften behandelten Geschäfte und Geheimnisse verschwiegen wäre. ${ }^{37}$

Überhaupt legte Martin Wert auf Geheimhaltung, und dies insbesondere bei der Arbeitsvermittlung: Niemand anderer als er sowie sein beschworner Gehülfe hätten Einblick in die Protokolle und auch den potenziellen Arbeitgebern würden die Namen der Arbeitssuchenden erst dann preisgegeben, wenn diese nach Informierung über die Qualifikation einer Person dem Fragamt explizit den Auftrag erteilt hätten, die betreffende Person zuzuschicken. ${ }^{38}$

Das Fragamt - dessen Standort innerhalb Pressburgs mir leider nicht bekannt ist - verfügte auch über ein Amts-Depositorium zur Lagerung der in ihm verkauften Waren, wozu Schmuck und Uhren ${ }^{39}$ genauso zählten wie Bücher, ${ }^{40}$ Mineralwasser, ${ }^{41}$ Antiwanzenmittel, ${ }^{42}$ Mottenpulver, Pillen gegen Zahnschmerzen, ${ }^{43}$ Senf, ${ }^{44}$ Tabak, ${ }^{45}$ Kippas - Kappeln, wie solche die Judenschaft zu tragen pflegetund Knöpfe. ${ }^{46}$ Auffallend ist, dass Martin mit den KäuferInnen via seines Kundschaftsblatts regelrecht kommunizierte: Als er einmal ankündigte, dass demnächst Marschansker Aepfel verkauft würden, informierte er potenzielle InteressentInnen, dass deren Ankunft und nächster Preis (...) auf einen geschriebenen Zettel an der äußern Amts-Thür zu sehen seyn würde. ${ }^{47}$ Speise- und Lampenöl wurde in Flaschen abgegeben, für die ein Einsatz von fünf Groschen zu bezahlen war; das Leergut wurde zurückgenommen: [D]iejenigen, so die Flaschen unbeschädigt, nebst denen Stoppeln zurück bringen, bekommen auch den 5ten Groschen wieder zurück. ${ }^{48}$ Zuweilen sind auch Rückkoppelungseffekte feststellbar: So war der im Fragamt verkaufte Fruchtsirup - hergestellt aus Ribisel-Erdbeeren bzw. Himbeeren - denen Liebhabern zu dick und zu süß; dem Produzenten wurde dies mitgeteilt, worauf dieser ihn dünner, und etwas ansäuerlich machte. ${ }^{49}$ Vermittelt wurden des weiteren Sonnenblumen- und Wassermelonenkerne: Als deren Erntezeit nahte, ließ Martin verlautbaren, dass das Fragamt bereit dazu war, diese, soferne sie denn gut ausgetrocknet waren, anzukaufen. ${ }^{50}$

Wenn es eine Ware gab, die wegging wie warme Semmeln, so war dies Wein, der mit vielen Beifall abgenommen wurde ${ }^{51}$ gleich von April 1781 an waren an Sorten Tokayer, Maßlasch, St. Georger Ausbruch und ordinarer St. Georger, Schomlauer, Rusther, Oedenburger, Ratzstorfer, von verschiedenen Alter, Qualität, Quantität, und Preisen zu haben, weiters war auch Sliwowitz im Angebot. Wer wollte, konnte davon im Fragamt auch verkosten. ${ }^{52}$ Die Weinbauern rief 
Martin via Kundschaftsblatt dazu auf, ihn wissen zu lassen, ob sie Wein flaschenweise vorrätig hätten und ob sie bereit wären, ihn mittels des Amts zu verkaufen, denn immer wieder hätten hiesige als auch reisende Personen bei ihm nachgefragt, wo man zum allenfalsigen Magen-Schluß, oder zum mitnehmen auf die Reise ein Glas guten, und gerechten Tokayer, Oedenburger, Rusther, Schomlauer, oder Ratzstorfer Wein bek[ä]me. ${ }^{53}$ Für diejenigen Herrschaften, die eine Tischgesellschaft mit Wein versorgen wollten, bot das Fragamt im Dezember 1782 als Service an, am Tag nach der Bewirtung die übrig gebliebenen, ungeöffneten Flaschen Wein zurückzunehmen; außerdem konnte Wein, der auf keinen Gefallen stie $\beta$, retourniert werden. ${ }^{54}$

Was die Wohnungsvermittlung anbelangt, so klagte Martin wiederholt darüber, dass zu wenig Wohnungseigentümer willens wären, leerstehende Wohnungen mittels des Fragamts anzubieten; häufig würden Wohnungssuchende beim Fragamt vorstellig, alleine, die Hausherren würden es zu ihren eigenen Schaden versäumen, dem Fragamte Nachricht von ihren zu verlassenden Wohnungen zu geben, wobei doch erst bei tatsächlicher Vermietung Vermittlungsgebühren anfallen würden, nicht aber jedoch - wie ursprünglich vorgesehen - für die reine Einschreibung und Bekanntmachung der leeren Wohnungen. ${ }^{55}$ Zunächst nämlich hatte der Fragamtsdirektor beginnend mit Juli 1781 folgendes Prozedere für die Wohnungsvermittlung vorgesehen: Die Hauseigentümer hatten eine je nach Höhe der Miete gestaffelte Einschreibegebühr von 3 bis 10 Kreuzer zu bezahlen, worauf die zu vermietende Wohnung in ein Protokoll eingetragen sowie im Kundschaftsblatt annonciert wurde; bezahlten die Eigentümer die doppelte Taxe, so wurde die Wohnung in ein weiteres Protokoll verzeichnet, das im Gegensatz zu dem ersten allen Wohnungssuchenden gegen eine Gebühr von 4 Kreuzern öffentlich zur Einsicht preis gegeben wurde. Die Bezahlung dieser doppelten Gebühr wurde laut Martin nur deswegen verlangt, damit Ordnung und Richtigkeit erhalten würden; würde eine protokollierte Wohnung vermietet und der Hausinhaber dies dem Amt anzeigen, würde diese aus dem Buch gestrichen werden und der Vermieter die zweite Taxe zurückerhalten. ${ }^{56}$ Es ging Martin also vorrangig um die Ajourhaltung seiner Protokolle; spätestens, als er den Zahlungsmodus für die Vermittlungsgebühr auf nachträgliche Bezahlung umstellte, war dieses Anliegen allerdings nicht mehr leicht zu verwirklichen: Nur zu oft sollte es dann vorkommen, dass wohnungssuchende Parteien zu einer Unterkunft gewiesen wurden, die bereits vermietet war, ohne dass dies dem Fragamt gemeldet worden war, ${ }^{57}$ nur selten konnte er im Kundschaftsblatt eine Nachricht wie diese einschalten, dass [d] as in vorgehenden Wochenblättern benannte Ungarisch-Altenburger Coffée-Haus (...) bereits verkauft worden sei. ${ }^{58}$ Der Direktor ersuchte die Vermieter daher, ihm wöchentlich oder zumindest vierzehntägig mitzuteilen, ob die eingetragene Wohnung bereits vermietet war oder nicht; war sie nicht vermietet, so war 
er bereit, sie nochmals unentgeltlich im Kundschaftsblatt zu annoncieren, was im übrigen auch für alle anderen zum Verkauf stehenden Waren galt. ${ }^{59}$

Vielerlei Vorteile hätte eine solche Benützung des Fragamts im Gegensatz zu den herkömmlichen Methoden der Wohnungssuche: Bislang wäre man oft einen ganzen Tag dabei herumgegangen und hätte an s.v. Schuhen dreymal so viel ab[ge]nutzet, als diese $4 \mathrm{kr}$. Taxe betragen würde; manch einer hätte sich müde gegangen, und die Zeit versplittert, welche vielleicht mit Erwerbung einigen Verdienstes hätte zurückgeleget werden können. Beim Fragamt wäre es demgegenüber nur nötig, die wenigen Schritte ins Amt zu gehen, dort in das Protokoll Einsicht zu nehmen und sodann ohne Umwege zum dort beschriebenen Haus zu gehen. Viele auf traditionelle Weise Wohnungssuchende würden zwar die an den Häusern angeschlagenen, zuweilen unscheinbaren oder verschmutzten Zettelchen sehen, wären aber oft nicht fähig, diese zu lesen und würden dann den Hausinhaber oder dessen Bedienstete unnütz strapazir[en], um dann erst recht zu erfahren, dass die angebotene Wohnung für sie bald zu groß, oder zu klein, oder zu theuer wäre. Auch käme vor, dass derlei Zettel öfters aus blosser Malitz eines Nachbars, oder eines Inwohners, oder auch aus Schelmerey der Jugend herabgerissen würden. Schließlich gäbe es noch einen weiteren Vorteil: Manchmal würden Quartiersuchende nach Vorfinden eines Zettels in ein Haus eintreten, aber den Hausmeister bzw. den Hausinhaber nicht auffinden und stattdessen einen vielleicht erbitterten Mieter vorfinden, der auf den Hausbesitzer nicht gut zu sprechen wäre und der zu vermietenden Wohnung allerlei Defekte andichten würde, wodurch das ganze Haus in üblen Ruf geraten würde. Das Fragamt würde hingegen den Quartier Suchenden directe an den Haus-Herren, Haus-Frau, oder Hausmeister anweisen, die ihn dann persönlich die Wohnung zeigen würden; der gehässig[e] Inwohner würde dann sich etwas moderiren, und mit schädlichen Verläumdungen etwas sparsamer seyn. ${ }^{60}$

$\mathrm{Zu}$ den weiteren Dienstleistungen des Fragamts zählte die Besorgung von Geschäften am Pressburger Versatzamt: Wer nicht selbst seine dort deponierten Pfänder auslösen wollte, konnte sich des Fragamts als Mittler bedienen, auf Wunsch auch anonym; es reichte, dem Fragamt ein versiegeltes Billet ohne Unterschrift zukommen zu lassen, in dem das Anliegen genau beschrieben wurde; mit pünktlichster Genauigkeit, dann strengster Verschwiegenheit würde der Wille des Einbringers befolgt werden. ${ }^{61}$ Das Fragamt wollte sich allerdings nicht nur darauf beschränken, Versatzamtsdienste zu vermitteln, sondern machte sich auch erbötig, selbst zum Ort von Versteigerungen zu werden, zumindest kündete Martin im August 1781 an, künftig Licitationen abhalten zu wollen. Personen, die willig waren, ihre Habe zur Versteigerung zu bringen, müssten dies nur schriftlich anzeigen, Martin würde darauf den Versteigerungstag publizieren lassen. An Taxen verrechnete er bei erfolgten Verkauf zwei Kreuzer vom Gulden; sollte die Ware nicht versteigert worden sein oder nicht den gewünschten Endpreis erzielt 
haben, wären zehn Kreuzer an Unkostenbeitrag zu bezahlen. ${ }^{62}$ Es ist nicht bekannt, ob derlei Versteigerungen tatsächlich stattfanden; ein halbes Jahr später wiederholte Martin jedenfalls sein Angebot. ${ }^{63}$

Eher schleppend scheint das Geschäft der Geldvermittlung angelaufen zu sein; als das Fragamt in einer Woche einmal mehrere Geldbedürftige, und mehrere Geld anzulegen gesonnene zu beyderseitig grosser Zufriedenheit zusammengebracht hatte, sprach Martin von einer gesegnete[n] Woche und verlieh seiner Hoffnung Ausdruck, dass das Amt beim Publikum noch mehr Vertrauen gewinnen und letzteres dem Vorurtheil nicht so viel Platz einräumen würde. ${ }^{64}$ Zuweilen bemühten sich auch Erfinder, mittels des Fragamts für die Verwirklichung ihrer Projekte Kapital aufzunehmen: So zeigte der berühmte Mechanikus Herr Musy an, dass er das unschätzbare Geheimniß erfunden habe, eine Mühle zu verfertigen, die nur durch einen Mühljungen oder mittels sehr wenig Kraft angetrieben werden konnte; er würde eine solche errichten, wenn Investoren eine Summe von 1000 Dukaten aufbrächten. ${ }^{65}$ Offensichtlich fanden sich einige Kapitalgeber, denn drei Wochen später vermeldete das Kundschaftsblatt, dass mehrere Personen dem Mühlen-Erfinder ein Douceur versprochen hätten und kommentierte dies mit dem Jubelschrei: Ein neuer Beweis der aufgeklärten Welte! ${ }^{66}$

Das Adressbüro fungierte weiters als Fundamt; nachdem ein gewisser Mathias Holler, ein gebohrner Deutschböhm, 32 Jahr alt, verheyrathet, folgsam glaubwürdigen Standes, ausgedienter herrschaftlicher Hausknecht, dermalen Diensttauglicher Sesselträger mit Nro. 28 auf dem Hut eine goldene Sackuhr gefunden und im Fragamt deponieren lassen hatte, fahndete Martin nach deren Besitzer: Wer selbe zu beschreiben im Stande ist, solle sie auf der Stelle als ihme zuerkennendes Eigenthum in seine Hände bekommen. ${ }^{67}$

Sorge bereitete Martin die hohe Mobilität seiner KlientInnen: Wiederholt kam es vor, dass diese einen Wohnungswechsel nicht beim Fragamt anzeigten, was zur Folge hatte, dass er InteressentInnen in die Irre schickte; er bat daher jedermann, der seine Wohnung veränder[n würde], solches anzudeuten, damit man es im Protokoll abänderen könne, massen es denen Partheyen selbst zum Nachtheil gereichet, wann man sie bey Vorfallenheiten nicht zu finden vermag. ${ }^{68}$ Überhaupt war das Fragamt auf die Personensuche spezialisiert und veröffentlichte im Kundschaftsblatt Aufrufe, den Wohnort von Personen unbekannten Aufenthalts zu melden; eine solche Annonce lautete z. B.: Eine sichere Elisabetha Schuberthin von Herren-Grund bey Neusohl gebürtig, so allhier in Preßburg als Zimmer-oder Stuben-Magd sich befinden solle, wird von ihren leiblichen Bruder gesucht. So jemand von dieser Elisabeth Schuberthin einige Wissenschaft ihres Aufenthalt-Orts haben sollte, der beliebe es dem Frag-Amt gütigst zu melden. ${ }^{69}$ Zumindest in einem dieser Fälle konnte Martin dann auch eine Erfolgsmeldung bringen, als nämlich einem Pressburger Handelsmann Post - ein Brief samt Paket - zugestellt worden war, die an eine Madame de Weinert née de Plecrer de Plan á Preszbourg 
adressiert waren; da alle Nachfrage ergebnislos war, wandte sich Martin mittels des Kundschaftsblatts an die Pressburger Öffentlichkeit ${ }^{70}$ und konnte tatsächlich schon eine Woche später zufrieden feststellen, dass die Post der Frau Eigenthümerinn zu ihrer nicht geringen Zufriedenheit richtig zugestellet und behändiget worden wäre. Der Fragamtsdirektor nahm dies zum Anlass mitzuteilen, daß alle Personen, deren Wohnungen unwissend sind, durch dieses Fragamt leicht aufgesucht und gefunden werden könn [t]en. ${ }^{71}$

Ein zusätzliches Angebot stand den BenützerInnen des Pressburger Fragamts spätestens ab Jänner 1782 zur Verfügung, nämlich das einer Leihbibliothek. Bereits im vorhergehenden Sommer hatte das Kundschaftsblatt angekündigt, dass eine ungenannte Person ein Ansuchen um Bewilligung eines Lesekabinetts nach Vorbild des Trattnerschen Lecturkabinetts zu Wien eingereicht hätte. Erwartet wurde, dass 100 Pränumeranten jährlich 20 Gulden für dessen Benützung zahlten; zu lesen geboten werden sollten ihnen deutsche, lateinische, ungarische, französische, italienische und englische Bücher. ${ }^{72}$ Dieser Plan eines Lesesaals scheint nicht verwirklicht worden zu sein; stattdessen bot Martin den lesewilligen PressburgerInnen ein halbes Jahr später an, im Fragamt Romane gegen die Gebühr eines Kreuzers pro Tag auszuleihen, ${ }^{73}$ bald standen auch Sachbücher zur Verfügung. ${ }^{74}$ Der Fragamtsdirektor beeilte sich mitzuteilen, dass keine etwa anstössige, oder gar unerlaubte Bücher ausgegeben würden, sondern nur solche, die die Zensur passiert hätten. ${ }^{75}$ Die Liste der zur Ausleihe angebotenen Bücher wurde zunächst im Kundschaftsblatt veröffentlicht; die Bücher waren nummeriert und deren höchste Nummer betrug 409, wobei es allerdings auch unnummerierte Bücher $\mathrm{gab}^{76}$ und Martin später konzedieren musste, dass der im Kundschaftsblatt zur Verfügung stehende Raum nicht mehr ausreichte, die Neuerwerbungen zu verzeichnen. Stattdessen versicherte er, dass im Fragamt die besten in- und ausländischen deutschen erlaubten Bücher von allerlei Inhalt, zum Lesen vorhanden wären. ${ }^{77}$ Sollte jemand ein Buch lesen wollen, das nicht auf dieser Liste verzeichnet war, so bestand die Möglichkeit, dieses für den dritten Teil seines Preises zu pränumerieren; das Buch wurde dann durch das Fragamt angeschafft und die betreffende Person konnte es so viele Tage ausleihen, als die Pränumeration Kreuzer betrug. Auf diese Weise könnte man ein Buch, das mehrere Gulden kostet, um einen Groschen lesen. ${ }^{78}$ Martin war sich auch bewusst, dass für Parteien, die außerhalb Pressburgs wohnten oder aber sich mit ausgeliehenen Büchern auf Reisen begeben wollten, die Leihgebühr von einem Kreuzer täglich zu teuer war; diesen bot er an, mehrere Bücher gegen Bezahlung eines Pauschalbetrags zu entlehnen oder sich zuschicken zu lassen. ${ }^{79}$ Manchmal kam es vor, dass die LeserInnen ihre Bücher gleich im Fragamt selbst zu lesen gewillt waren, was allerdings nicht auf Gegenliebe der Fragamtbetreiber stieß: Letztere baten die Herrn Liebhaber ergebenst, sich mit Lesen der Bücher im Amte selbst nicht zu beschäftigen, da sie da- 
durch in ihren Geschäften aufgehalten würden und dies außerdem jenen Fremden, die etwa etwas Geheimes anzubringen h[ätt]en, unangenehm wäre. ${ }^{80}$

Im Falle eines äußerst wertvollen Buchs, nämlich Lavaters Physiognomischen Fragmenten, hatte sich Martin ein besonderes Prozedere ausgedacht: Anfang September 1782 kündigte er an, dass das Fragamt dieses Werk, dessen Kosten von 160 Gulden einem Privatmann die Lektüre fast verunmöglichen würden, anzuschaffen gedachte, es müssten sich nur 50 bis 60 Interessenten finden, die zur Bezahlung von 2 Gulden bereit wären. An diese wollte Martin Billets ausgeben, die in der Reihenfolge der einlangenden Pränumerationen nummeriert sein sollten; die vier Teile des Lavaterschen Werks würden in zwölf Bände gegliedert, und der Pränumerant mit der Nummer 1 könnte den ersten Band ab 1. Oktober 1782 für drei Tage zur Lektüre entlehnen, woraufhin er am 3. Oktober den zweiten Band bekäme und der Pränumerant mit dem Billet Numero 2 nunmehr den ersten Band erhalten würde. Selbstverständlich war dieses ausgeklügelte System davon abhängig, dass die strengste Genauigkeit beobachte[t] wurde; es ist nicht bekannt, ob es tatsächlich realisiert wurde. ${ }^{81}$

Zunächst waren es nur Bücher, die über das Fragamt entlehnt werden konnten; das recht zeitgleich mit der Eröffnung der Leihbibliothek von einem Freund vorgetragene Ansinnen, eine Zeitungslesegemeinschaft zum Bezug der Wiener und der Erlanger Zeitung zu gründen, scheint sich mangels Interesse bald zerschlagen zu haben. ${ }^{82}$ Als der Fragamtsdirektor Anfang Juni 1782 dafür warb, in- und ausländische Zeitschriften und Zeitungen zu subskribieren, ${ }^{83}$ stieß dies ebenfalls auf wenig Resonanz: Interessenten hätten dafür jährlich sieben Gulden zu zahlen gehabt und die betreffenden Periodika jeweils zwei Tage entlehnen können, ${ }^{84}$ alleine, es fanden sich viel zu wenig Liebhaber, was Martin allerdings nicht davon abhalten sollte, trotzdem zehn bis zwölf Journale zu bestellen. ${ }^{85}$ Noch Ende 1783 vermeldete das Kundschaftsblatt, dass im Fragamt künftig gegen monatliche Pränumeration von 34 Kreuzern auch Zeitschriften zu entlehnen wären. ${ }^{86}$ Die Inhaltsverzeichnisse mancher der bestellten Zeitschriften wurden im Kundschaftsblatt veröffentlicht, das somit auch die Funktion eines Referatediensts übernahm. ${ }^{87}$

Welche Aufgaben übernahm das Fragamt noch? - Es nahm Anfragen um Reisebegleitung entgegen, die nicht nur im Medium des Kundschaftsblatts veröffentlicht wurden, sondern auch per Anschlagszettel an den äusseren Amts- und Gassenthüre $[n] ;{ }^{88}$ immer wieder brachte das Kundschaftsblatt Annoncen von Eltern, die ihre Kinder - Knaben und Mägdlein - als so genannte Tauschkinder zum Fremdspracherwerb für einige Zeit in einer anderssprachigen Familie unterbringen wollten: [K]ein bequemeres Mittel würde es geben, daß die Kinder mit geringeren Unkösten entweder ihre Mutter, oder eine andere Sprache erlernen könn [t]en, ${ }^{89}$ alleine, Martin bedauerte, daß hiesigen Innwohnern dieser Antrag nicht gefallen wollte, noch dazu, wo die betreffenden Kinder nicht von Bauersleu- 
ten, sondern von Kondition wären. ${ }^{90}$ Und Personen, die zeichnen lernen wollten, verlieh das Fragamt Zeichnungen als Vorlage. ${ }^{91}$

Das Pressburger Frag- und Kundschaftsamt sollte nur knapp drei Jahre Bestand haben; bereits im September 1783 teilte Martin seinem Publikum mit, dass er das Kundschaftsblatt mit Ende des Monats schließen wollte. ${ }^{92}$ Er entschloss sich dann - auf Ratschlag seiner KundInnen - doch, das Blatt in abgespeckter Form noch einige Wochen weiterzuführen, ${ }^{93}$ bis er dann mit Ende des Jahrs das endgültige Aus des Kundschaftsblatts - und damit wohl auch des Fragamts - verkündete; er begründete dies mit dem Umstand, dass der Mangel an Materie immer größer würde. ${ }^{94}$ Vielleicht gab es in Pressburg mit seinen gerade mal 30.000 EinwohnerInnen tatsächlich nicht genügend Kundschaft für ein Adressbüro; eventuell spielte auch eine Rolle, dass Pressburg in eben dem Jahr 1783 seine Hauptstadtfunktion an Buda verlor. ${ }^{95}$ Insgesamt scheint das Pressburger Fragamt keine größere Rolle im Leben der Stadt gespielt zu haben; in Johann Matthias Korabinskys Beschreibung der königl. ungarischen Haupt- Frey- und Krönungsstadt Preßburg wird es nicht erwähnt und weder Friedrich Nicolai noch Heinrich Sander, die 1781/82 Pressburg besuchten, schenkten dieser Einrichtung Beachtung. ${ }^{96}$ Bemerkenswert an der Martinschen Einrichtung erscheint die Vielfalt der dargebotenen Dienstleistungen, deren Spektrum von der Hilfestellung beim profanen Austausch alltäglicher Güter bis hin zum Angebot einer Leihbibliothek reichte; es war fürwahr Anspruch des Pressburger Fragamts, Stätte universeller Vermittlung zu sein.

\section{Anmerkungen}

1

Die vorliegende Arbeit entstand im Rahmen eines vom österreichischen Fonds zur Förderung der wissenschaftlichen Forschung (P 19826-G08) unterstützten Forschungsprojekts. Ich bedanke mich bei Dorottya Lipták, Ilona Pavercsik und Jozef Tancer für wertvolle Informationen und bei Erika Szőke (Kathedralbibliothek Esztergom) für die Übersendung von Scans des Jahrgangs 1783 des Preßburger Kundschaftsblatts.

2 E-mail: anton.tantner@univie.ac.at; Postadresse: Institut für Geschichte, Universität Wien, Dr. Karl Luegerring 1, 1010 Wien, Österreich. Homepage: http://tantner.net

3 Allgemein zu den Adressbüros siehe Blome, Astrid: Vom Adressbüro zum IntelligenzblattEin Beitrag zur Genese der Wissensgesellschaft. - In: Jahrbuch für Kommunikationsgeschichte 8 (2006), S. 3-29; zum 1630 gegründeten Pariser Bureau d'Adresse siehe: Solomon, Howard M.: Public Welfare, Science and Propaganda in seventeenth Century France: The Innovations of Théophraste Renaudot. Princeton: Princeton UP, 1972; Feyel, Gilles: L'Annonce et la nouvelle. La presse d'information en France sous l'ancien régime (1630-1788). Oxford: Voltaire Foundation, 2000, S. 11-308; Stagl, Justin: Eine Geschichte der Neugier. Die Kunst des Reisens 1550-1800. Wien et. al.: Böhlau, 2002, S. 175-190; Jubert, Gérard (Hg.): Père des journalistes et médecin des pauvres. Théophraste Renaudot (1586-1653). Paris: Champion, 2005. 
$4 \quad \mathrm{Zu}$ diesem Themenbereich organisierten Thomas Brandstetter, Thomas Hübel (Institut für Wissenschaft und Kunst) und ich im Oktober 2008 in Kooperation mit der Wienbibliothek im Rathaus das Symposion „Vor Google. Suchmaschinen im analogen Zeitalter“; Tagungshomepage mit Programm: http://www.univie.ac.at/iwk/vor-Google/ Tagungsbericht: http://hsozkult.geschichte.hu-berlin.de/tagungsberichte/id=2446 (letzter Zugriff jeweils 4.4.2011); eine Publikation im Transcript-Verlag ist für 2012 in Vorbereitung. Als erste Annäherung an eine solche Vorgeschichte siehe auch: Tantner, Anton: Suchen und Finden vor Google. Eine Skizze. - In: VÖB-Mitteilungen 64 (2011), Heft 1, 41-68 (in Druck).

5 Als Skizze zu den habsburgischen Fragämtern siehe: Tantner, Anton: Frag- und Kundschaftsämter in der Habsburgermonarchie als Institutionen der Informations- und Wissensvermittlung. - In: Frimmel, Johannes; Wögerbauer, Michael (Hg.): Kommunikation und Information im 18. Jahrhundert. Das Beispiel der Habsburgermonarchie. Wiesbaden: Harrassowitz, 2009 (=Buchforschung. Beiträge zum Buchwesen in Österreich 5). S. 309-320. Ein umfassender Artikel zum Wiener Fragamt ist von mir zur Publikation in den Wiener Geschichtsblättern eingereicht; zu den Brünner und Prager Fragämtern bereite ich zur Zeit eine Veröffentlichung für die Zeitschrift Folia Historica Bohemica vor.

6 Der Portheimkatalog der Wienbibliothek im Rathaus kennt mehrere Personen dieses Namens: Einen Kaufmann Anton Martin (geboren um 1758, gestorben in Prag am 5.7.1818), einen Arzt gleichen Namens, gestorben in Ybbs am 27.1.1819, weiters Anton Freiherr Martin (um 1760 Wien, Stock im Eisen Nr. 623, 11.3.1839), 1822 Direktor des geheimen Kabinetts des Kaisers sowie den mährischen Guberniumskanzlisten Anton Wilhelm Martin, 1789 Mitglied der Brünner Loge; bei letzteren handelt es sich vermutlich um dieselbe Person: Er war auch gemeinsam mit Anton Franz Schrötter Adjunkt des Bücherrevisionsamts in Brünn. Wiener Zeitung, 15.3.1839, S. 378; D'Elvert, Christian: Beiträge zur Geschichte und Statistik Mährens und Oesterreichisch-Schlesiens. 1. Bd.: Geschichte des Bücher- und Steindruckes, des Buchhandels, der Bücher-Censur und der periodischen Literatur (...). Brünn: Rohrer, 1854 (= Schriften der historisch-statistischen Sektion der k.k. mähr. schles. Gesellschaft des Ackerbaues, der Natur- und Landeskunde 6). S. 155; D'Elvert, Christian: Die Freimaurer in Oesterreich, besonders Mähren. - In: Notizen-Blatt der historisch-statistischen Section der kais. königl. mährisch-schlesischen Gesellschaft zur Beförderung des Ackerbaues, der Naturund Landeskunde. (Beiblatt zu: Mittheilungen der Kaiserlich-Königlichen, MährischSchlesischen Gesellschaft zur Beförderung des Ackerbaues, der Natur- und Landeskunde in Brünn), 1866, Nr. 1, S. 1-6, hier 6; D’Elvert, Christian: Zur Oesterreichischen Verwaltungsgeschichte mit besonderer Rücksicht auf die böhmischen Länder. Brünn: Winiker, 1880 (=Schriften der historisch-statistischen Section der k.k. mähr.-schles. Gesellschaft zur Beförderung des Ackerbaues, der Natur- und Landeskunde 24). S. 617; Trautenberger, Gustav: Die Chronik der Landeshauptstadt Brünn. 4. Bd.: Vom Beginn des 18. Jahrhunderts bis zur Auflösung des römischen Reichs deutscher Nation. Brünn: Deutsches Haus, 1897, S. 178. Laut Auskunft von Anna Buzinkayová (E-Mail vom 2.10.2009) haben sich im Archív hl. mesta SR Bratislavy keine Akten zu Martin erhalten. 1785 arbeitete Martin im Lecturkabinet in Ofen: Weiner Zeitung, 13.8.1785, S. 1913.

7 Avertissement, 9.4.1781, Beilage zu: Wiener Zeitung, Nr. 40, 19.5.1781, [unpaginiert], Signatur: ÖNB 1,005.524-D/1780, 1. Bd. Die Beilage ist weder in der Mikrofilm- noch (zur Zeit) in der Onlineversion von ANNO erfasst.

8 Zum Tauschkindersystem siehe u.a.: Kósa, László: Kinderaustausch und Spracherlernen in Ungarn. - In: Hungarian Studies 3 (1987), Heft 1-2, S. 85-93; Paládi-Kovács, Attila: Kindertausch und interethnische Kontakte im Karpatenbecken. - In: Bringéus, Nils-Arvid et al. (Hg.): Wandel der Volkskultur in Europa. Festschrift für Günter Wiegelmann zum 60. 
Geburtstag. Münster: Coppenrath, 1988, Bd. 1, S. 271-279; Liszka, József: Das TauschkindSystem im slowakischen Teil der Kleinen Tiefebene. - In: Zeitschrift für Balkanologie 32 (1996), S. 58-72; Ötvös, Péter: Deutschlernen in Preßburg. - In: Kriegleder, Wynfrid; Seidler, Andrea; Tancer, Jozef (Hg.): Deutsche Sprache und Kultur im Raum Pressburg. Bremen: Edition lumière, 2002 (=Presse und Geschichte - Neue Beiträge 4). S. 17-26; Liszka, József: Kinderaustausch als Methode des Fremdsprachenerwerbs. - In: Ders.: Volkskunde der Ungarn in der Slowakei. Zwischen den Karpaten und der Ungarischen Tiefebene. Passau: Lehrstuhl für Volkskunde der Universität, 2003 (=Passauer Studien zur Volkskunde 22). S. 219-238. Vgl. auch Fielhauer, Helmut Paul: Kinder-,,Wechsel“ und „Böhmisch-Lernen “. Sitte, Wirtschaft und Kulturvermittlung im früheren niederösterreichisch-tschechoslowakischen Grenzbereich. - In: Österreichische Zeitschrift für Volkskunde 81, N.S. XXXII (1978). S. 115-148. Avertissement, 9.4.1781, Beilage zu: Wiener Zeitung, Nr. 40, 19.5.1781, [unpaginiert].

10 Preßburger Kundschaftsblatt (PK), III. Stück 15.-21.4.1781, S. 12; IV. Stück, 22.-28.4.1781, S. 16. Die Jahrgänge 1781-1782 dieser Publikation befinden sich in der Univerzitná knižnica v Bratislave, einige Ausgaben des Jahrgangs 1783 in der Főszékesegyházi Könyvtár.

11 Avertissement, 9.4.1781, Beilage zu: Wiener Zeitung, Nr. 40, 19.5.1781, [unpaginiert].

12 PK, IV. Stück, 22.-28.4.1781, S. 16.

13 PK, XII. Stück, 3.6.-9.6.1781, S. 45.

14 PK, VII. Stück, 13.5.-19.5.1781, S. 25.

15 PK, VIII. Stück, 20.5.-26.5.1781, S. 33.

16 PK, XXIII. Stück, 2.9.-8.9.1781, S. 100.

17 PK, XVIII. Stück, 29.7.-4.8.1781, S. 70.

18 PK, XVI. Stück, 15.7.-21.7.1781, S. 61.

19 PK, XXXVIII. Stück, 15.9.-21.9.1782, S. 150.

$20 \quad$ PK, XLIII. Stück, 20.10.-26.10.1782, S. 171.

21 PK, III. Stück 15.-21.4.1781, S. 9f; VII. Stück, 13.5.-19.5.1781, S. 27; XIII. Stück, 24.6.-30.6.1781, S. 52; 1783 konnten manche Ausgaben dann bis zu 20 Seiten umfassen.

22 PK, V. Stück, 29.4.-5.5.1781, S. 19f.

23 PK, XVII. Stück, 22.-28.7.1781, S. 67.

24 PK, XXII. Stück, 26.8.-1.9.1781, S. 87.

25 PK, XII. Stück, 3.6.-9.6.1781, S. 45.

26 PK, Nr. 38 20.12.1783, S. 456.

27 PK, XXVI. Stück, 24.6.-30.6.1782, S. 102.

28 PK, XLV. Stück, 3.11.-9.11.1782, S. 178.

29 PK, XII. Stück, 3.6.-9.6.1781, S. 45.

$30 \quad$ PK, Nr. 13, 28.6.1783, S. 197.

31 PK, V. Stück, 29.4.-5.5.1781, S. 19.

32 PK, VI. Stück, 6.5.-12.5.1781, S. 22.

33 PK, V. Stück, 29.4.-5.5.1781, S. 19.

34 PK, XXXIV. Stück, 18.11.-24.11.1781, S. 146.

35 PK, XXXVIII. Stück, 16.12.-22.12.1781, S. 162.

36 PK, XIV. Stück, 31.3.-6.4.1782, S. 53; Wiederholung im XV. Stück, 7.4.-13.4.1782, S. 57f, umfangreiche Preisliste auf S. 59.

37 PK, III. Stück, 13.1.-19.1.1782, S. 9.

38 PK, XII. Stück, 3.6.-9.6.1781, S. 45.

39 PK, V. Stück, 29.4.-5.5.1781, S. 17f.; VIII. Stück, 20.5.-26.5.1781, S. 30.

$40 \quad$ PK, XXV. Stück, 17.6.-23.6.1782, S. 96.

41 PK, XII. Stück, 3.6.-9.6.1781, S. 47. 


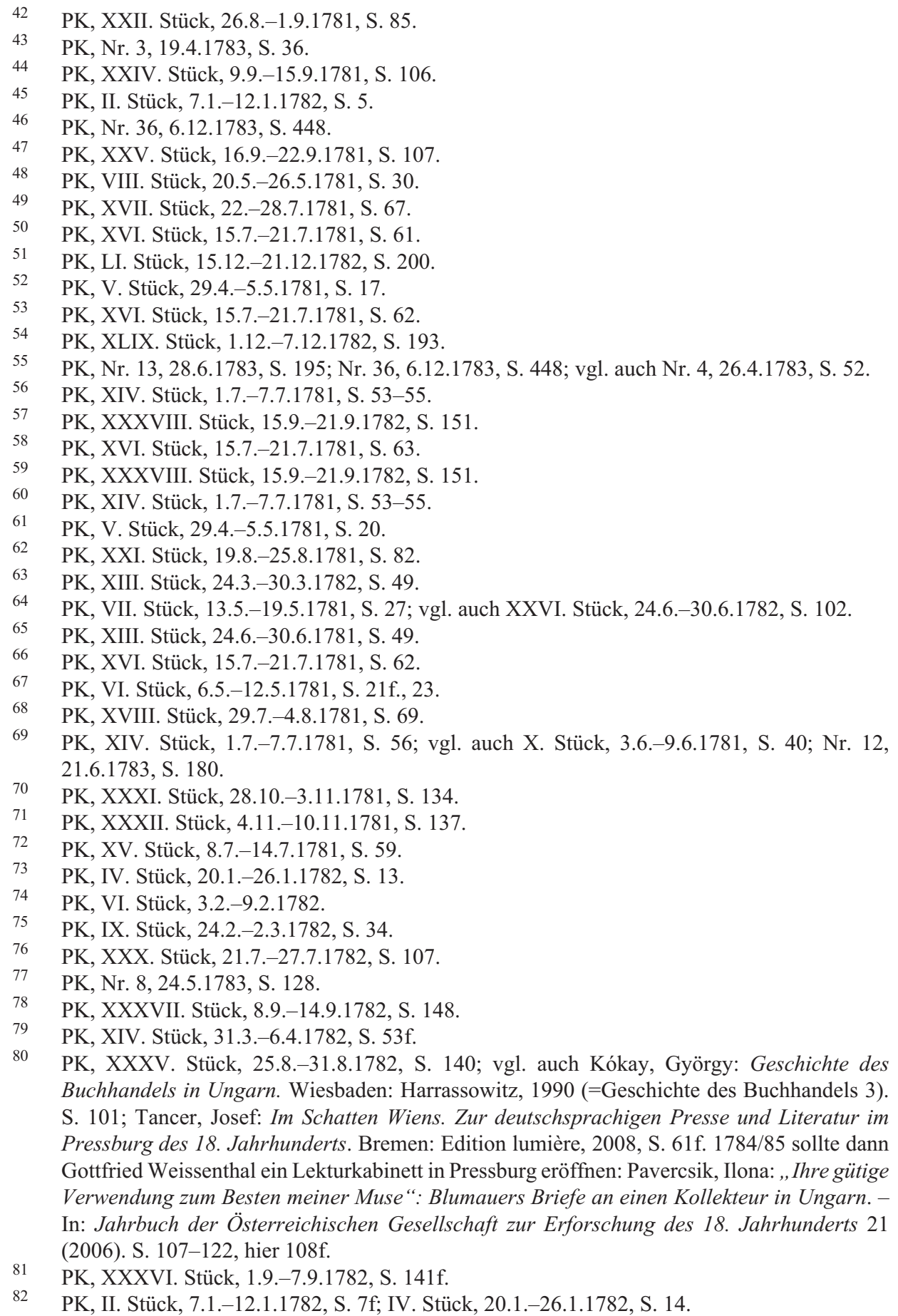


PK, XXIII. Stück, 2.6.-8.6.1782, S. 90 f.

84 PK, XXIV. Stück, 9.6.-16.6.1782, S. 95; vgl. auch XXV. Stück, 17.6.-23.6.1782, S. 98; XXX. Stück, 21.7.-27.7.1782, S. 119; XLI. Stück, 6.10.-12.10.1782, S. 163; XLII. Stück, 13.10.-19.10.1782, S. 163f.; XLVI. Stück, 10.11.-16.11.1782, S.184.

85 PK, XLIX. Stück, 1.12.-7.12.1782, S. 196.

86 PK, Nr. 37, 13.12.1783, S. 451; Nr. 38, 20.12.1783, S. 456.

87 PK, XXXVIIII. Stück, 22.9.-28.9.1782, S. 155f., vgl. auch u.a. XLI. Stück, 6.10.-12.10.1782, S. 164.

88 PK, IX. Stück, 27.5.-2.6.1781, S. 33.

89 PK, X. Stück, 3.6.-9.6.1781, S. 39.

90 PK, XIX. Stück, 14.10.-20.10.1781, S. 124; vgl. I. Stück, 1.-7.4.1781, S. 2; Nr. 27, 4.10.1783, S. 411 ; Nr. 30, 25.10.1783, S. 423.

$91 \quad$ PK, VI. Stück, 3.2.-9.2.1782, S. 22.

92 PK, Nr. 25 20.9.1783, S. 392 (statt falsch 390).

93 PK, Nr. 26 27.9.1783, S. 401, 408.

94 PK, Nr. 39 27.12.1783, S. 460.

95 Allgemein zur Situation Pressburgs im 18. Jahrhundert siehe: Tancer, Schatten, S. 21-65.

96 Korabinsky, Johann Matthias: Beschreibung der königl. ungarischen Haupt- Frey- und Krönungsstadt Preßburg. Preßburg: Korabinsky, o.J., 1781; Nicolai, Friedrich: Beschreibung einer Reise durch Deutschland und die Schweiz, im Jahre 1781. Berlin/Stettin 1783. ND Hildesheim u.a.: Olms, 1994. (=Gesammelte Werke 16. Hg. von Fabian, Bernhard; Spieckermann, Marie-Luise); Sander, Heinrich: Heinrich Sanders (...) Beschreibung seiner Reisen durch Frankreich, die Niederlande, Holland, Deutschland und Italien; in Beziehung auf Menschenkenntnis, Industrie, Litteratur und Naturkunde insonderheit. 2 Bände. Leipzig: Friedrich Gotthold Jacobäer und Sohn, 1783-1784. 